\title{
EPES comprehensive evaluation model of grain system based on principal component analysis
}

\author{
Zuyi Zhang, Yipu Cao, Kaishuo Liu* \\ School of Economics, Minzu University of China, Haidian, Beijing, 100081, China \\ *Corresponding Author
}

\begin{abstract}
The establishment of a coordinated food system is the basis for supporting economic development, protecting the natural environment and maintaining social achievements. In our paper, three countries with large grain output and rich grain system -- the United States, India and Brazil were selected as the research objects. Firstly, the EPES comprehensive evaluation model of grain system based on principal component analysis and analytic hierarchy process was established to realize the comprehensive evaluation of four subsystems of grain system at three national scales. The sensitivity analysis of the consumer price index in the evaluation model is also carried out.
\end{abstract}

Keywords: food system optimization, sustainable development, principal component analysis.

\section{Introduction}

Food is foundation of the healthy development of human society. The current food system, which ensures efficiency and profitability, is controlled by a few large countries or companies, this profit-based system contributes to the inequity and unsustainability of food production and consumption globally and within a given country. The global outbreak of Covid-19 has severely impacted the fragile global agrifood system, which reminds us that the task of re-optimizing the future food system is urgent.

The future food system should achieve high efficiency and high yield, low nutrition and carbon, strong sustainability and equity. Equity and sustainability play an important role in coping with various shocks. They are important means to help people, communities, countries and global institutions prevent, predict, prepare, respond to and recover from shocks and become richer. In this paper, the evaluation model of food system are established to optimize the food system, which is expected to increase the fairness and sustainability of the global food system.

\section{EPES Comprehensive Evaluation model}

\subsection{Evaluation framework}

1) Determination of the scope of the food system assessment

The food system includes many links from food production to food waste treatment. In order to make the efficiency, profitability, equality and sustainability clear, the key statistics are related indicators of the links of food production and consumption, it's a simplification of the real food system.

2) Determination of food system classification

The food system is divided into two categories. Food systems in developed countries use data from the United States, and those in developing countries use data from Brazil and India. The three countries all rank high in grain output, and they all belong to the major grain exporting countries.

3) Direction of food system assessment

According to the requirements, the priority of food system is divided into four categories: high efficiency system, high profit system, high equity system and high sustainability system. Based on total $\mathrm{CO} 2$ emissions from agricultural land, grain production, average dietary energy adequacy, total grain production index and per capita agricultural value added, current food system priorities will be judged. 


\subsection{Analytic hierarchy process}

\subsubsection{Indicators affecting the food system}

Through PCA, the linear combination of the original index was found and the index affecting the food system of the countries concerned was obtained. In the process of dimensionality reduction, it is assumed that the selected index basically contains most of the important information of the original index, and the influence of other non-principal components on data analysis can be ignored. Data from the United States, India and Brazil for 2008-2017 were selected and averaged. After normalization, Table 1 was obtained as the basis for analysis.

Table 1: Food system indicators and data for the three countries

\begin{tabular}{|c|c|c|c|}
\hline Indicator & US & India & Brazil \\
\hline CPI & 0.3175 & 0.3212 & 0.3613 \\
\hline TCEA & 0.9962 & 0.0036 & 0.0002 \\
\hline GY & 0.9134 & 0.0466 & 0.0400 \\
\hline ADEA & 0.3800 & 0.2774 & 0.3427 \\
\hline TGPI & 0.3256 & 0.3433 & 0.3311 \\
\hline GCPI & 0.3223 & 0.3426 & 0.3352 \\
\hline PAVA & 0.8790 & 0.0151 & 0.1059 \\
\hline GA & 0.7726 & 0.2170 & 0.0104 \\
\hline
\end{tabular}

\subsubsection{Selection and analysis of principal component indicators}

According to the data in Table 1, factor analysis was carried out with SPSS, and the corresponding coefficients were obtained, and the indexes were normalized. Then the main components are selected.

After calculation, we can know that the ratio of principal component 1 is $79.60 \%$, and that of principal component 2 is $20.39 \%$. The total ratio of the two is $100.00 \%$. Therefore, principal component 1 and principal component 2 can be used to represent all the indicators. The coefficient corresponding to the index is obtained by the interpretation of composition matrix and total variance.

Table 2: Composition coefficient

\begin{tabular}{|c|c|c|c|c|c|c|c|c|}
\hline Category & $I A_{1}$ & $I A_{2}$ & $I A_{3}$ & $I A_{4}$ & $I A_{5}$ & $I A_{6}$ & $I A_{7}$ & $I A_{8}$ \\
\hline Principal component 1 & -0.174 & 0.392 & 0.391 & 0.342 & -0.330 & -0.387 & 0.396 & 0.363 \\
\hline Principal component 2 & -0.704 & 0.117 & 0.121 & -0.397 & 0.434 & 0.172 & 0.407 & 0.314 \\
\hline
\end{tabular}

Table 3: Results of the normalization of each indicator

\begin{tabular}{cccc}
\hline Category & US & India & Brazil \\
\hline CPI & -0.65255 & -0.49873 & 1.15128 \\
TCEA & 1.15470 & -0.57438 & -0.58032 \\
GY & 1.15467 & -0.57064 & -0.58404 \\
ADEA & 0.89803 & -1.07763 & 0.17960 \\
TGPI & -0.85613 & 1.09909 & -0.24296 \\
GCPI & -1.07692 & 0.89927 & 0.17764 \\
PAVA & 1.14941 & -0.67028 & -0.47913 \\
GA & 1.11438 & -0.29523 & -0.81915 \\
\hline
\end{tabular}

After the above steps, the score $F_{1}$ of principal component 1 , the score $F_{2}$ of principal component 2 and the linear combination $\mathrm{F}$ of the original index can be calculated. Take the US:

$$
\begin{gathered}
\mathrm{F}_{1}=0.174 \mathrm{ZX}_{1}+0.392 \mathrm{ZX}_{2}+0.391 \mathrm{ZX}_{3}+0.342 \mathrm{ZX}_{4}+0.330 \mathrm{ZX}_{5}+0.387 \mathrm{ZX}_{6}+0.396 \mathrm{ZX}_{7}+0.363 \mathrm{ZX}_{8}=2.89 \\
\text { Again, } F_{2} \text { is } 0.62 \\
\mathrm{~F}=\frac{80}{100} F_{1}+\frac{20}{100} F_{2}=2.44
\end{gathered}
$$

Calculate $F_{1}, F_{2}$ and $\mathrm{F}$ for each of the three countries to arrive at the final conclusion, as shown in Table 4: 
Academic Journal of Computing \& Information Science

ISSN 2616-5775 Vol. 4, Issue 4: 51-55, DOI: 10.25236/AJCIS.2021.040409

Table 4: Final results of principal component analysis

\begin{tabular}{ccccc}
\hline Country & $F_{1}$ & $F_{2}$ & $\mathrm{~F}$ & Rank \\
\hline US & 2.89 & 0.62 & 2.44 & 1 \\
Brazil & -1.09 & -1.54 & -1.18 & 2 \\
India & -1.77 & 0.46 & -1.32 & 3 \\
\hline
\end{tabular}

On the basis of the conclusions reached, a reasonable inference is made that $F_{1}$ is the total emission of $\mathrm{CO}_{2}$ from agricultural land and $\mathrm{F}_{2}$ is the grain yield.

\subsubsection{Construction of tomographic structural model}

According to the relationship among the goal of the decision, the factors to be considered and the decision objects, the hierarchical structure chart is drawn. Using the results of principal component analysis and reasonable judgment, the indexes of $F_{1}, F_{2}, F_{3}, F_{4}$ and $F_{5}$ are determined, which are total $\mathrm{CO}_{2}$ emission, the grain yield, and the average dietary energy supply adequacy, the total grain production index and per capita agricultural value added; B1, B2, B3 and B4 stand for efficient systems, high profit systems, equity systems, and sustainability systems, respectively.

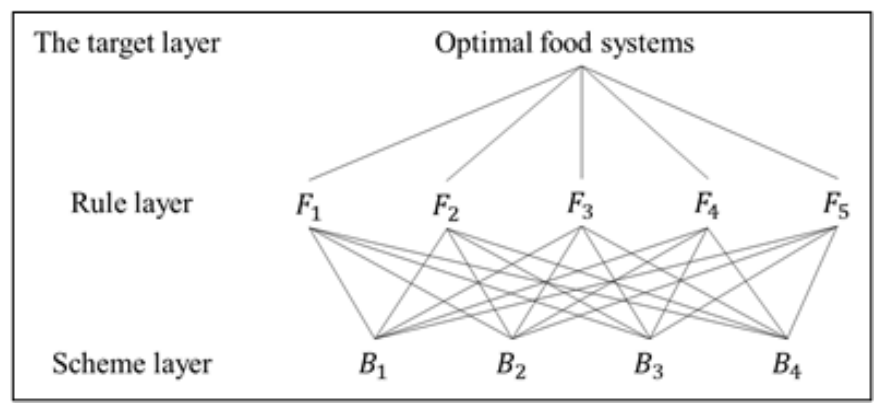

Figure 1: Schematic diagram of the hierarchical model

A judgment matrix is a comparison that indicates the relative importance of all factors in the hierarchy to one factor in the hierarchy above. A total of five evaluation factors were rated by 10 experts from demography, environment and the agricultural economics on the basis of scientific principles and practical factors using the Saaty scale of $1-9$, resulting in the following pairwise comparison matrix.

$$
A=\left[\begin{array}{ccccc}
1 & 0.5 & 2 & 2 & 0.5 \\
2 & 1 & 5 & 3 & 5 \\
0.5 & 0.2 & 1 & 0.3 & 0.5 \\
0.5 & 0.3 & 3 & 1 & 1 \\
2 & 0.2 & 2 & 1 & 1
\end{array}\right]
$$

\subsection{Implementation}

\subsubsection{Hierarchical single sort and consistency checking}

We define consistency metrics as follows.

$$
\mathrm{CI}=\frac{\lambda-\mathrm{n}}{\mathrm{n}-1}
$$

By introducing the random consistency index RI, we define the consistency ratio:

$$
\mathrm{CR}=\frac{\mathrm{CI}}{\mathrm{RI}}
$$

Generally, when the consistency ratio $\mathrm{CR}$ is less than 0.1 , it is considered that the degree of inconsistency of A is within the allowable range and there is satisfactory consistency, that is, it passes the consistency test.

The maximum eigenvalue of pairwise comparison matrix $\mathrm{A}$ is $\lambda=5.270$

The normalized eigenvectors of this eigenvalue are $\omega=\{1.280,2.150,0.370,0.697,0.503\}$ 
Then $\mathrm{Ci}=0.068, \mathrm{RI}=1.120, \mathrm{CR}=0.060<0.1$, indicating that a passed the consistency test.

\subsubsection{Hierarchical summation sort and consistency checking}

The maximum eigenvalue of matrix $B_{1}$ is $\lambda_{1}=4.071$, and the eigenvector corresponding to this eigenvalue is $\omega_{1}=\{0.484,1.667,1.078,0.771\}$.Similarly, the maximum eigenvalues and eigenvectors of $B_{2}, B_{3}, B_{4}$ and $B_{5}$ can be obtained, as shown in Table 5 .

Table 5: Calculation results of Tier 3 versus Tier 2

\begin{tabular}{c|c|c|c|c|c}
\hline$\omega_{2}$ & 1.280 & 2.150 & 0.370 & 0.691 & 0.503 \\
\hline \multirow{3}{*}{$\omega_{3}$} & 0.512 & 0.370 & 2.223 & 0.245 & 0.311 \\
\cline { 2 - 5 } & 1.525 & 2.326 & 1.014 & 2.136 & 2.086 \\
\cline { 2 - 5 } & 1.075 & 0.622 & 0.352 & 0.791 & 0.722 \\
\cline { 2 - 5 } & 0.887 & 0.681 & 0.411 & 0.828 & 0.880 \\
\hline$\lambda$ & 4.218 & 4.046 & 4.155 & 4.179 & 4.133 \\
\hline CI & 0.073 & 0.015 & 0.052 & 0.060 & 0.044 \\
\hline RI & 0.890 & 0.8900 & 0.89 & 0.890 & 0.890 \\
\hline CR & 0.082 & 0.017 & 0.058 & 0.067 & 0.050 \\
\hline
\end{tabular}

$\mathrm{Ci}, \mathrm{Ri}$ and $\mathrm{CR}$ all pass the consistency test, so the final combination weight of different systems can be calculated. The specific results are as follows.

The combined weight of option $B_{1}$ against the objective is:

$$
0.512 \times 1.280+0.370 \times 2.150+2.223 \times 0.370+0.245 \times 0.691+0.311 \times 0.503=0.357
$$

In the same way, the combination weight of Scheme $B_{2}, B_{3}, B_{4}$ and $B_{5}$ is $0.165,0.176,0.225,0.077$ respectively. Therefore, the combination weight vector of the scheme layer to the target is $(0.375,0.165,0.176,0.225,0.077)^{T}$, which can be used as the final decision-making basis. The order of weight of each scheme is $B_{1}>B_{4}>B_{3}>B_{2}>B_{5}$, so the best choice is $B_{1}$. This suggests that the efficient system is the choice that best represents the overall picture of the current food system, namely that the current food systems in all three countries are biased towards high-efficiency systems.

\subsubsection{Sensitivity analysis}

In the analytic hierarchy process (AHP) model, the consumer price index (CPI) has an effect on principal component 1 and principal component 2, which affects the final score of the linear combination. It is necessary to know the extent to which changes in the consumer price index affect the final food system, and thus whether it is necessary to change the consumer price index at a greater cost. So we do a sensitivity analysis of the consumer price index.

In the solution, the three consumer price indices we choose are normalized and distributed in [-1,1.2]. In order to understand the effects of the consumer price index on the principal components 1 and 2, we first set $I A_{2}-I A_{8}$ to be 1 , then we set $I A_{1}$ to be $-2,-1,0,1,2$,orderly.Then,we calculate the corresponding principal component 1 and principal component 2 and draw a diagram comparing them as follows.

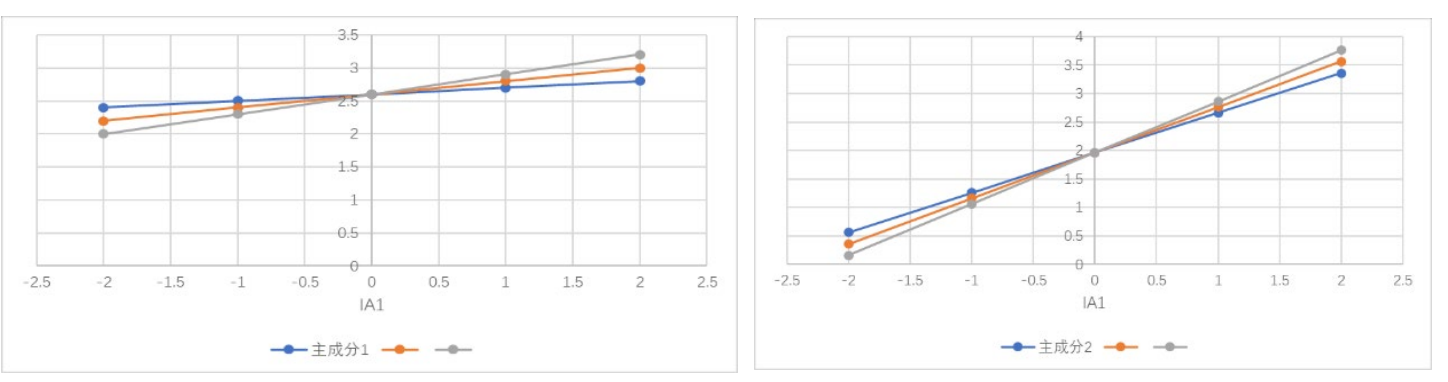

Figure 2

The image above is drawn with a $I A_{1}$ coefficient of $0.1,0.2,0.3$ and $0.7,0.8,0.9$, respectively. The graph above shows the following.

The sensitivity of the CPI is related to the coefficient, and the greater the preference coefficient of a country to the $\mathrm{CPI}$, the greater the influence of $I A_{1}$ on the overall final score $\mathrm{F}$.

The sensitivity of the consumer price index is independent of the location range, and the principal 
component 1 and principal component 2 increase with the increase of IA. At any point in the consumer price index range, $I A_{1}$ has the same effect on the final score of $\mathrm{F}$.

\section{References}

[1] Stecchini M L, Torre M D. The Food Safety Management System [J]. Veterinary Research Communications, 2005, 29 Suppl 2(s2): 117.

[2] Masiero, Silvia. Redesigning the Indian Food Security System through E-Governance: The Case of Kerala [J]. World Development, 2015, 67: 126-137.

[3] Morris K. US food-safety system needs major overhaul [J]. The Lancet, 1998, 352(9129): 716.

[4] Ayalew H. Review on food safety system: Ethiopian perspective [J]. African Journal of Food Science, 2013, 7(12): 431-440. 